\title{
Building structures within opal photonic crystals
}

Rudolf Zentel, Birger Lange, Shalin J. Jhaveri, and Christopher K. Ober

Engineered defects enhance control over light in photonic crystals. Two novel methods fabricate defects inside polymer opal photonic crystals.

The unique abilities of photonic crystals (PCs) to manipulate light lead to potential applications ranging from simple optical switches to an optical computer. ${ }^{1}$ Researchers are learning how to build PCs with better optical abilities by methods that include designing and fabricating deviations from the regular PC pattern. These deviations-called defects, although they are desirable - can channel light efficiently, but building defects in the structure is challenging. We have developed several methods for defining defect patterns inside of synthetic opal.

Opals are naturally occurring 3D PCs: their microstructure consists of silica spheres about $150 \mathrm{~nm}$ to $300 \mathrm{~nm}$ in diameter, which are tightly packed into repeating hexagonal or cubic arrangements. Synthetic opals use this same pattern, although they can be made from different materials. A type of PC made using the the gaps between the spheres is called an inverse opal. Self-assembly methods allow us to make large polymer opals and inverse opals at low cost.

Several research groups are focusing on fabricating complex 3D architectures in opals. David Norris at the University of Minnesota recently used multiphoton polymerization with a laserscanning confocal microscope to tailor air defects in opals. ${ }^{2}$ Also, George Zhao at the National University of Singapore described using an advanced photolithographic process to embed line defects in opals. ${ }^{3}$ We are developing two additional methods: using UV light to pattern the surface as the structure is built up; ${ }^{4}$ and using two-photon lithography to photopolymerize a resin inside an inverse opal structure, then writing embedded 3D patterns. ${ }^{5}$

The first method is analogous to patterning photoresists for photolithography, and offers the advantage of patterning the whole opal at once. In combination with a multilayer build-up method or with two-photon lithography, this scheme could even pattern the opal in 3D. ${ }^{5}$ To explain this method, we need to describe some of the steps and chemistry involved in making and

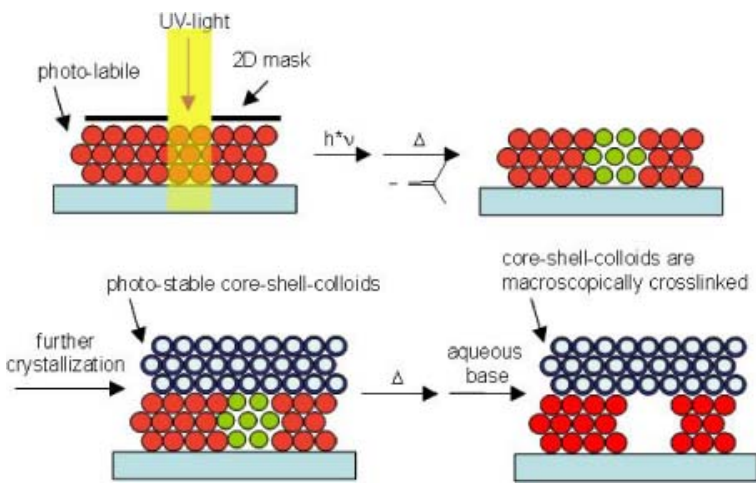

Figure 1. UV light through a mask forms 3D defects inside photonic crystals during a multilayer growth process. Where crystallized photolabile colloids (red dots) are exposed to UV light, they form a soluble acid (green dots). After photo-stable core-shell-colloids form another layer of crystal, the exposed material can be washed out using an aqueous base. The top layer can be thermally crosslinked.

patterning a 2D polymer opal. First, we synthesize photoprocessable beads in order to introduce defects via UV lithography. A major advantage of polymer beads is chemical flexibility. In this case, we use t-butyl methacrylate (tBMA) as the monomer because the t-butyl ester group can be cleaved under acidic conditions. Next, we convert the monomer into monodisperse spheres using surfactant-free emulsion polymerization. Then, the light-sensitive system-consisting of a photo-acid generator (PAG) and a sensitizing dye-is incorporated into the preformed colloids. At this point, the beads crystallize into polymer opals. Next, we expose part of the surface to UV light. During the optical exposure, the tBMA units change to methacrylic acid (MA) and release isobutene. Also, the PAG forms acid, which catalyzes an ester cleavage during the post-exposure bake. At the end of the process, we can selectively wash out the exposed area using an aqueous base.

We gain selective control of the third dimension by combining the chemistry (see Figure 1). We start by crystallizing a

Continued on next page 


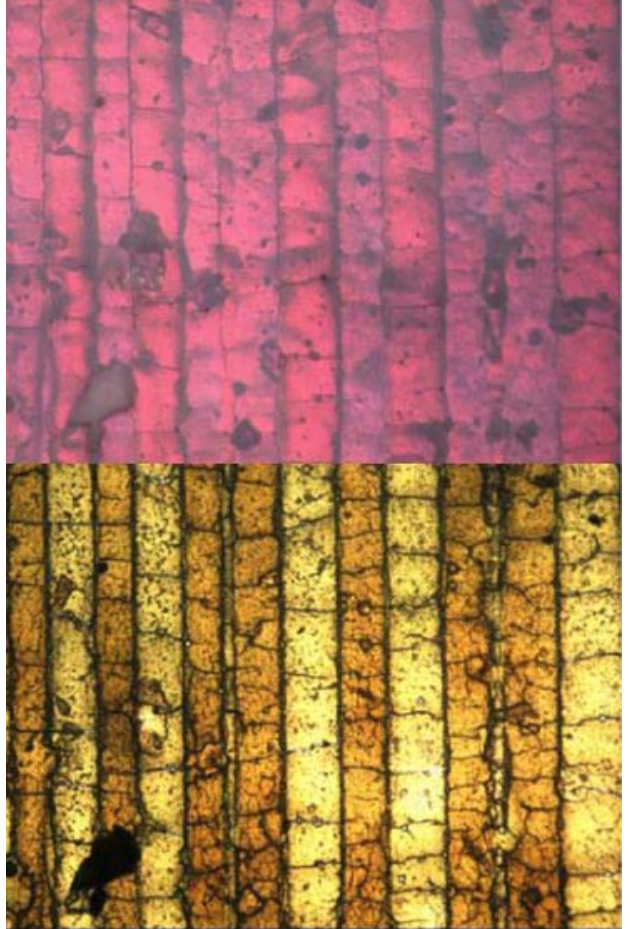

Figure 2. An optical microscope image shows the surface structure (top) and the bulk structure (bottom) of an opaline film after $3 D$ defect engineering.

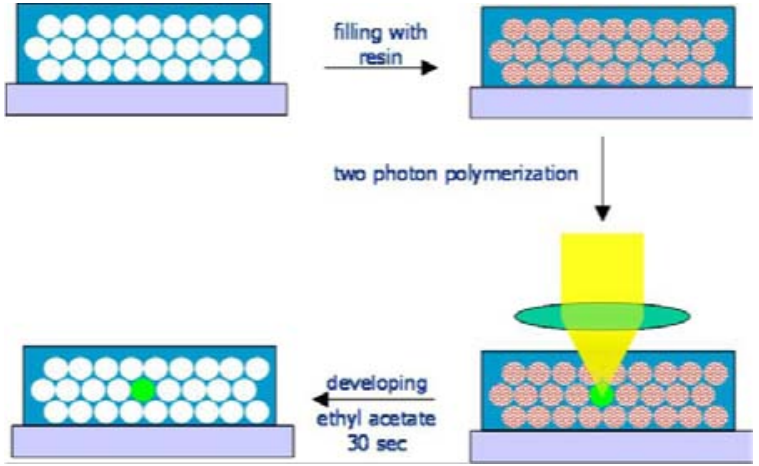

Figure 3. A defect can be formed in an inverse-opal photonic crystal by first filling the voids with a photo-polymerizable resin. The twophoton process only polymerizes resin at the focus, allowing defects to be created inside the material.

layer of photosensitive polymer beads. This layer is processed as described in the previous paragraph. Instead of completing the process and removing the exposed material, however, we crystallize a second layer of photo-stable beads on top of the first layer. At this point, the exposed areas of the photo-labile layer can be developed to create a defect within the volume of the opal

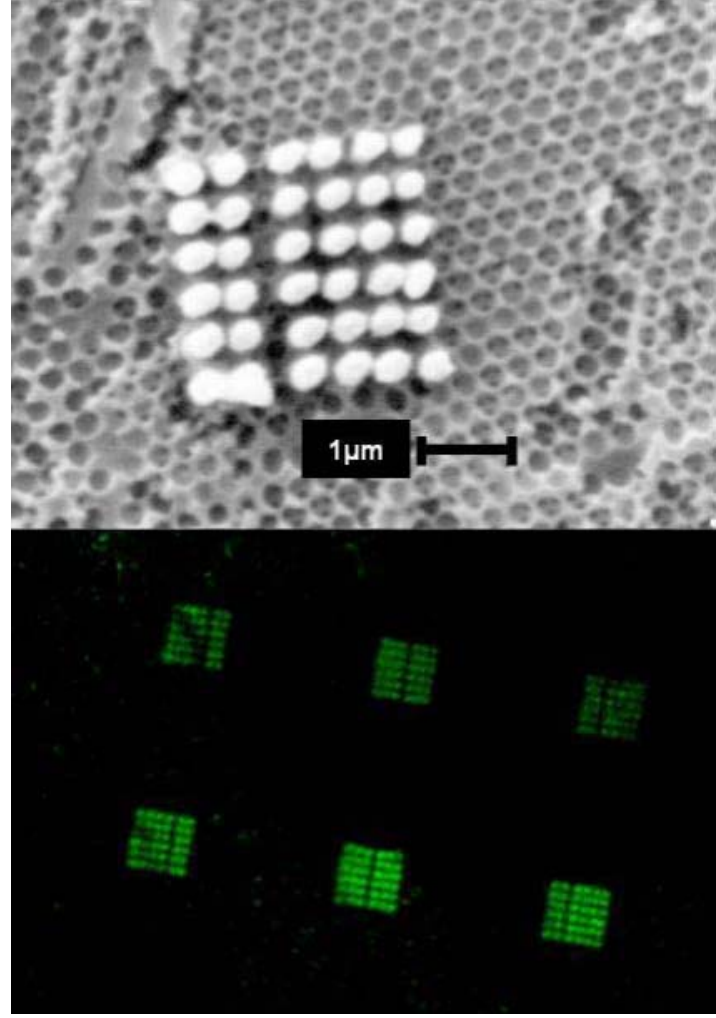

Figure 4. Two views of fluorescent defect structures in an inverse opal: the top image was obtained from a scanning electron microscope; the bottom image was captured by a fluorescence microscope.

(see Figure 2). This method provides structures with a resolution of several micrometers.

The second method we mentioned for incorporating 3D defects inside an opaline structure uses two-photon lithography. We can control all three dimensions of our structures by using a photopolymerizable resin in which a fluorescent dye is dissolved. With this method we managed incorporation of both emitters and defects in an inverted polymer opal (see Figure 3).

We made several different structures using two-photon lithography. Because a crucial benefit of two-photon lithography is the ability to detect fabricated structures-especially when they are embedded in a matrix as in our case- we first patterned the top layer and top of the inverse opal (see Figure 4). Subsequently, we fabricated defects at defined places inside the replica (see Figure 5). This allowed us to make structures with sub-micrometer resolution.

These two new methods allow us to fabricate 3D defects embedded in an opaline matrix. Both take advantage of colloidal self-assembly to form large periodic structures. In both methods, we use a multistep process to introduce defects. We are now

Continued on next page 


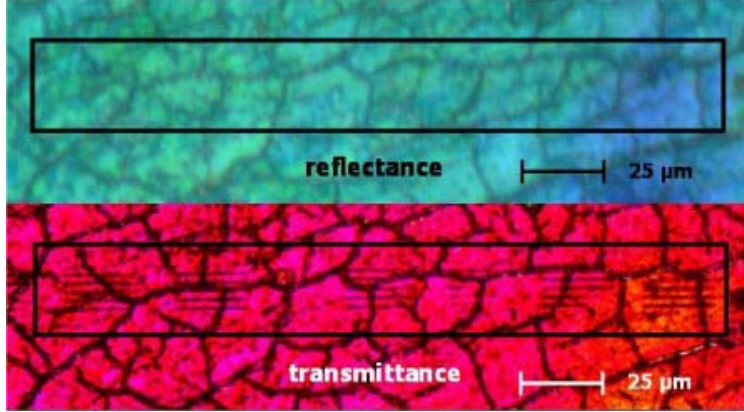

Figure 5. Optical microscope images of embedded line defects in a replica are invisible in the reflected-light image (top), but can be seen as thin dark lines when imaged with transmitted light (bottom).

considering ways to combine them-such as using photo-labile beads for two-photon-lithography-to simplify the process. If we could eliminate the need for multilayer crystallization, then we could potentially produce $3 \mathrm{D}$ defects directly inside the opal.

The authors of this paper would like to thank the DFG (Priority program: Photonic crystals) and the EU (PHAT) for financial support. We also thank the Cornell Center for Materials Research for partial support of this work.

\section{Author Information}

\section{Rudolf Zentel and Birger Lange}

Institute of Organic Chemistry

University of Mainz

Mainz, Germany

Rudolf Zentel is a professor of organic and polymer chemistry at the University of Mainz. His current research interests include photonic crystals, liquid crystals, semiconducting polymers, and multilayer systems.

Birger Lange is currently working towards a PhD at the Institute of Organic and Macromolecular Chemistry at the University of Mainz under the supervision of Rudolf Zentel. The subject of his thesis is the structuring of polymer opal photonic crystals.

\section{Shalin J. Jhaveri and Christopher K. Ober}

Department of Materials Science and Engineering

Cornell University

Ithaca, NY

Shalin Jhaveri is currently working towards a $\mathrm{PhD}$ at the Department of Materials Science and Engineering at Cornell
University. His supervisor is Christopher Ober. His research interests are hydrogels and two-photon lithography.

Christopher $\mathrm{K}$. Ober is a professor of materials science and engineering at Cornell University. His research involves the preparation of tailored polymer structures with specific molecular architectures for predefined properties.

\section{References}

1. J. D. Joannopoulos, R. D. Mead, and J. N. Winn, Photonic Crystals: Molding the Flow of Light, Princeton Univ. Press, 1995.

2. Y. Jun, C. A. Leatherdale, and D. J. Norris, Tailoring air defects in selfassembled photonic bandgap crystals, Adv. Mater. 17 (15), pp. 1908-1911, 2005 doi:10.1002/adma.200500026

3. Q. Yan, Z. Zhou, X. S. Zhao, and S. J. Chua, Line defects embedded in three-dimensional photonic crystals, Adv. Mater. 17 (15), pp. 1917-1920, 2005 doi:10.1002/adma.200500047

4. B. Lange, R. Zentel, C. K. Ober, and S. Marder, Photoprocessable polymer opals, Chem. Mater. 16 (25), pp. 5286-5292, 2004. doi:10.1021/cm048834u

5. B. Lange, R. Zentel, S. J. Jhaveri, and C. K. Ober, 3D defect engineering in polymer opals, Proc. SPIE 6182, 2006. doi:10.1117/12.662046 